\title{
Diabetes Insípido em Paciente com Esclerose Múltipla
}

\section{apresentação de caso}

\author{
FERNANDA G. WEILER \\ KÁtIA BLUMBERG \\ ClaUdia S. LIBONI \\ EDUARDO A. C. ROQUE \\ AÉCIO F. T. DE GÓls
}

Disciplina de Medicina de Urgência, Universidade Federal de São Paulo (Unifesp), SP, Brasil.
A esclerose múltipla (EM) é uma doença crônica e progressiva que se caracteriza por surtos de desmielinização que podem atingir qualquer topografia do cérebro, medula espinhal e nervo óptico. Sendo o diabetes insípido (DI) central causado, principalmente, em virtude de danos do sistema nervoso central (tais como trauma, cirurgia, tumor, infecção, sarcoidose), a EM está inclusa entre suas possíveis etiologias. Entretanto, a ocorrência dessa associação não é comumente descrita. A suspeita clínica deve ser feita na presença de poliúria e polidipsia ou hipernatremia refratária (em pacientes privados do acesso à água) durante a evolução da EM. Descreveremos um caso em que essa associação ocorreu e, após o início da terapêutica com desmopressina, a paciente reverteu o quadro clínico. (Arq Bras Endocrinol Metab 2008;52/1:134-137)

Descritores: Diabetes insípido; Esclerose múltipla; Hipernatremia; Desmopressina

\section{ABSTRACT}

\section{Diabetes Insipidus in a Pacient with Multiple Sclerosis.}

Multiple Sclerosis (ME) is a chronic progressive disease characterized by relapses of demyelination that can occur anywhere in the brain stem, spinal cord and optic nerve. Since central diabetes insipidus (DI) is mainly caused by central nervous system damage (such as trauma, surgery, tumor, infection, sarcoidosis), ME is included among its possible etiologies. However, this association is not commonly described. The clinical suspicion must be made in the presence of polyuria and polydipsia or refractory hypernatremia (in patients without free access to water) during the evolution of ME. We will describe a clinical report in which this association occurred and, after the beginning of desmopressin therapy, the clinical findings were reverted. (Arq Bras Endocrinol Metab 2008;52/1:134-137)

Keywords: Diabetes insipidus; Multiple sclerosis; Hypernatremia; Desmopressin

\section{INTRODUÇÃO}

\begin{abstract}
ESClerose Múltipla (EM) é uma doença que cursa com diversos sinais e sintomas neurológicos. Entretanto, o diabetes insípido (DI) central não é uma manifestação freqüentemente encontrada. Descreveremos, a seguir, o caso de uma paciente portadora de EM que desenvolveu DI central. Após o diagnóstico e adequado tratamento com desmopressina (DDAVP), um análogo do hormônio antidiurético (ADH ou vasopressina), a paciente evoluiu com resolução completa do distúrbio hidroeletrolítico.
\end{abstract}




\section{RELATO DE CASO}

Paciente do sexo feminino, 32 anos, branca, portadora de esclerose múltipla secundariamente progressiva há dez anos, apresentava como seqüela da doença bexiga neurogênica e déficit de força em membro inferior esquerdo. Procurou serviço médico por vômitos e dor abdominal, sendo diagnosticada infecção de trato urinário com evolução para choque séptico e necessidade de intubação orotraqueal. À sondagem vesical, houve diurese de 9 litros em 12 horas e conseqüente hematúria ex vaccum, sendo iniciada irrigação vesical contínua. Durante a internação, evoluiu com níveis crescentes de sódio sérico, atingindo valor máximo de $180 \mathrm{mEq} / \mathrm{L}$, apesar de adequada reposição volêmica com soro hipotônico. Em virtude da irrigação vesical, a quantificação da diurese não vinha sendo adequadamente realizada; entretanto, após minucioso controle, verificou-se volume urinário superior a 4 litros por dia. Outros exames: glicemia de jejum, magnésio e cálcio séricos normais; potássio, $3,2 \mathrm{mEq} / \mathrm{L}$, (normal: 3,5 a $5,0 \mathrm{mEq} / \mathrm{L}$ ); creatinina, $3,4 \mathrm{mg} / \mathrm{dL}$, (normal: 0,6 a $1,0 \mathrm{mg} / \mathrm{dL}$ ); uréia, $70 \mathrm{mg} / \mathrm{dL}$, (normal: 10 a $50 \mathrm{mg} / \mathrm{dL}$ ). Não foi possível dosar a osmolaridade urinária em virtude da irrigação vesical. Realizado teste com desmopressina (DDAVP), $0,1 \mathrm{~mL},(10 \mu \mathrm{g})$ intranasal e observada queda da diurese 12 horas após $(1,4 \mathrm{~L})$ em relação à diurese 12 horas prévias à administração da droga $(2,6 \mathrm{~L})$. Administrouse, então, $0,1 \mathrm{~mL}$ duas vezes ao dia com queda progressiva da concentração de sódio sérico, respeitando a variação máxima de $8 \mathrm{mEq} /$ dia. Mantida tal dose, a paciente vem apresentando concentrações de sódio entre 140 e $146 \mathrm{mEq} / \mathrm{L}$ e diurese média de $2 \mathrm{~L} /$ dia.

A ressonância nuclear magnética (RNM) de sela túrcica, realizada após a melhora clínica da paciente, não foi conclusiva para o diagnóstico (Figura 1).

\section{DISCUSSÃo}

A EM é uma doença crônica e potencialmente incapacitante que se caracteriza por inflamação, desmielinização e lesão axonal (1), atingindo cérebro, medula espinhal e nervo óptico. Afeta principalmente mulheres (relação 2:1) entre a $3^{a}$ e a $4^{a}$ décadas de vida (2). Acredita-se que seja uma desordem imunomediada que ocorre em indivíduos geneticamente susceptíveis (3); entretanto, o mecanismo deflagrador da doença permanece incerto (4).

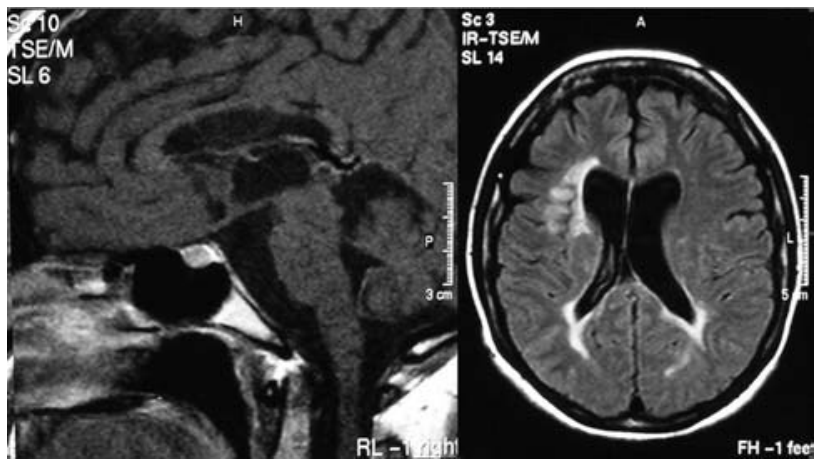

Figura 1. Ressonância nuclear magnética de sela túrcica mostrando presença de hipersinal em 11 na neuro-hipófise.

A EM produz uma gama de sinais e sintomas neurológicos dependendo dos locais anatômicos envolvidos. Os sinais sensitivos são a forma de apresentação mais comum (5). Outras alterações incluem fadiga; sintomas motores (fraqueza e espasticidade); incoordenação; anormalidades visuais (diminuição da acuidade visual, diplopia, presença de escotomas); disfunção vesical, intestinal e sexual; alterações cognitivas (2).

A doença é classificada de acordo com a apresentação inicial e sua forma de progressão. A forma mais comum é a surto-remissiva, presente inicialmente em 85\% dos casos (6). É caracterizada como dois ou mais surtos com duração maior que 24 horas e intervalo mínimo de 30 dias entre elas (1). Alguns desses pacientes, geralmente após 10 a 20 anos, evoluem com piora progressiva dos sintomas neurológicos, ainda que surtos possam ocorrer (7). Esse grupo é classificado então como forma secundariamente progressiva. Quinze por cento dos pacientes apresentam a forma primariamente progressiva, que se caracteriza por sintomas neurológicos progressivos ao longo de, no mínimo, seis meses (4).

O diagnóstico é eminentemente clínico. É preciso evidenciar lesões em diferentes locais do sistema nervoso central (SNC) em diferentes momentos da evolução (4), além de excluir outros diagnósticos. Exames complementares podem ser úteis, principalmente a RNM. Tal exame é um método de alta sensibilidade que, em portadores da doença, evidencia lesões multifocais hiperintensas em T2 (8). Os locais de maior especificidade são corpo caloso, tronco cerebral, substância branca periventricular e área justacortical (2). Lesões em atividade apresentam realce após a infusão de contraste (8). Nos casos duvidosos, podemos ainda lançar mão de outros exames, como a análise do líquido cerebroespinhal, que geralmente apresenta pleocitose linfo- 
cítica moderada, aumento de imunoglobulina G e/ou presença de bandas oligoclonais. Os estudos de potencial evocado permitem evidenciar lesões subclínicas (4).

O tratamento da crise baseia-se principalmente no uso de corticosteróides e a terapia a longo prazo é realizada com quimioterápicos, interferon e anticorpos monoclonais, entre outros (2). Nenhuma forma de tratamento trouxe resultados plenamente satisfatórios. A terapêutica está voltada para atenuar o processo inflamatório.

A DI é uma desordem caracterizada por perda de grandes volumes de urina (poliúria) hipotônica, geralmente acompanhada de polidipsia. Poliúria é definida como diurese superior a 3 litros em 24 horas (9). Há dois diferentes mecanismos causadores da doença. $\mathrm{O}$ primeiro é resultado da diminuição da resposta das células renais ao $\mathrm{ADH}$, caracterizando a DI nefrogênica. Já a chamada DI central é causada pela incapacidade de secreção de $\mathrm{ADH}$ pela hipófise posterior (e/ou déficit de produção pelo hipotálamo) em resposta ao aumento da osmolaridade sérica (10).

A DI central ocorre igualmente em ambos os sexos, com idade de início entre 10 e 20 anos na maioria dos casos (9). Suas principais causas são danos ao sistema nervoso central (trauma, cirurgia, tumor, infecção, sarcoidose, histiocitose etc.), genética (destruição da glândula) ou idiopática (11). Poliúria e polidipsia definem o quadro clínico. Somente se o paciente estiver inconsciente ou incapaz de reconhecer a sensação de sede ocorrerá hipernatremia e desidratação (12). A história clínica e os exames complementares específicos são úteis para o diagnóstico diferencial de poliúria.

Os métodos diagnósticos variam conforme a literatura estudada. Pode-se dosar a osmolaridade urinária, cujo valor deverá ser abaixo de $200 \mathrm{mOsm} / \mathrm{kg}$ e a osmolaridade plasmática, que poderá ter seus níveis acima dos considerados normais. É possível também realizar o teste de privação de água ou o teste da vasopressina. Este é realizado, entre outras maneiras, administrandose 0,05 a $0,1 \mathrm{~mL}$ de DDAVP intranasal ou $1 \mu \mathrm{g}$ subcutâneo ou intravenoso e observando-se a variação do volume urinário 12 horas antes e 12 horas após a administração. Indivíduos portadores de DI central apresentarão redução importante da diurese (13). Definido o diagnóstico de DI central, a realização de RNM de crânio poderá evidenciar diferentes alterações de acordo com cada etiologia (14).

O tratamento da DI central consiste na correção dos distúrbios hidroeletrolíticos e do déficit de água. A droga de escolha é o DDAVP. Pode ser administrado por via parenteral oral ou intranasal, e a dose deve ser individualizada. Outras drogas podem ser utilizadas em situações especiais como clorpropramida, carbamazepina, tiazídicos e indometacina (9).

Descrevemos o caso de uma paciente do sexo feminino com diagnóstico de EM secundariamente progressiva que evoluiu com hipernatremia refratária à correção com soro hipotônico. Devemos destacar que a paciente encontrava-se inconsciente e, portanto, incapaz de reconhecer a sensação de sede. O diagnóstico de poliúria foi retardado pela presença de irrigação vesical contínua, o que também impediu a realização da dosagem de osmolaridade urinária. Diante das dificuldades de execução de tal exame e do quadro crítico com risco iminente de morte causado pela hipernatremia grave, optamos por realizar prontamente o teste com vasopressina. Observamos queda importante da diurese e da concentração de sódio sérico, levando-nos ao diagnóstico de DI central.

A imagem da RNM não nos ajudou no diagnóstico de DI neste caso, pois a neuro-hipófise normal pode apresentar hipersinal em Tl, assim como as lesões desmielinizantes inflamatórias da EM na fase aguda. Logo, o diagnóstico nesta fase é clínico. Com o decorrer do tempo, ocorre perda deste hipersinal, a hipófise se torna hipointensa e o diagnóstico de DI pode ser confirmado radiologicamente.

A importância do caso descrito advém da raridade de relatos na literatura mundial de DI causada por EM (15-17).

\section{REFERÊNCIAS}

1. Bruck W, Stadelmann C. Inflammation and degeneration in multiple sclerosis. Neurol Sci. 2003;24(5):265-67.

2. Fox RJ, Bethoux F, Goldman MD, Cohen JA. Multiple Sclerosis: Advances in understanding, diagnosing, and treating the underlying disease. Cleve Clin J Med. 2006;73(1):91-102.

3. Hohlfeld R. Biotechnological agents for the immunotherapy of multiple sclerosis: principles, problems and perspectives. Brain. 1997;120:865-916.

4. Noseworthy JH, Locchinetti C, Rodriguez M, Weinshenker BG. Mulltiple Sclerosis. N Engl J Med. 2000;343(13):938-52.

5. Poser S, Wikstrom J, Bauer HJ. Clinical data and the identification of special forms of multiple sclerosis in 1271 cases studied with a standardized documentation system. J Neurolol Sci. 1979;40:159-68.

6. Lublin FD, Reingold SC. Defining the clinical course of multiple sclerosis: results of an international survey. Neurology. 1996;46:907-11. 
7. Weinshenker BG, Bass B, Rice JPA, et al. The natural history of multiple sclerosis: a geographically based study. I. Clinical course and disability. Brain. 1989;112:133-46.

8. Pyhtinen J, Karttunen A, Tikkakoski T. Increasing benefit of magnetic resonance imaging in multiple sclerosis. Acta Radiol. 2006;47(9):960-71.

9. Makaryus AN, McFarlane SI. Diabetes insipidus: Diagnosis and treatment of a complex disease. Cleve Clin J Med. 2006;73(1):65-71.

10. Singer I, Oster JR, Fishman LM. The management of diabetes insipidus in adults. Arch Intern Med. 1997;157:1293-1301.

11. Adam P. Evaluation and management of diabetes insipidus. Am Fam Physician. 1997;55:2146-53.

12. Buonocore $\mathrm{CM}$, Robinson AG. The diagnosis and management of diabetes insipidus during medical emergencies. Endocrinol Metab Clin North Am. 1993;22(2):411-23.

13. McPhee SJ, Papadakis MA, Tierney LM. Diseases of the Hypothalamus \& Pituitary Gland. Current Medical Diagnosis \& Treatment 2007. $46^{\text {th }}$ edition: $1132-33$
14. Shin JH, Lee HK, Choi CG, Suh DC, Kim CJ, Hong SK, et al. MR Imaging of central diabetes insipidus: A pictorial essay. Korean J Radiol. 2001;2(4):222-30.

15. Mathon K. On centrally-conditioned endocrine disorders in multiple sclerosis. (Temporary occurrence of diabetes insipidus). Psychiatr Neurol Med Psychol. 1994:137-9.

16. Voiculescu V, Psapta DM. Diabetes insipidus in a patient with suspected multiple sclerosis. Rom J Neurol Psychiatry. 1990;28(2):153-4.

17. Maghnie M, Genovese E, Bernasconi S, Binda S, Arico M. Persistent High MR Signal of the Posterior Pituitary Gland in Central Diabetes Insipidus. Am J Neuroradiol. 1997;18:1749-52.

\section{Endereço para correspondência:}

Fernanda Guimarães Weiler Rua Napoleão de Barros, 715 04024-002 São Paulo, SP

E-mail: fegweiler@yahoo.com.br 